\title{
Unilateral Multifocal Intraocular Lens Implantation in a Patient with Adie"s Pupil
}

\author{
Stephen A. LoBue ${ }^{a}$ Fukutaro Mano ${ }^{b}$ Erin Schaefer ${ }^{a}$ Thomas D. LoBue \\ aLoBue Laser and Eye Medical Centers, Inc., Murrieta, CA, USA; ${ }^{b}$ Suita Tokushukai Hospital \\ Eye Center, Suita, Japan
}

\section{Keywords}

Multifocal intraocular lens $\cdot$ ReSTOR $\cdot$ Adie's pupil $\cdot$ Tonic pupil

\begin{abstract}
Purpose: To report a case of a patient with unilateral Adie's pupil who underwent bilateral cataract extraction with multifocal and monofocal posterior chamber intraocular lens (IOL) implantation. Methods: A 74-year-old woman presented to our institution complaining of worsening near vision. Gross examination revealed a $6-\mathrm{mm}$ fixed pupil on the right eye (OD) and a 5 - $\mathrm{mm}$ pupil reacting to $3 \mathrm{~mm}$ with light on the left eye (OS). Slit lamp examination revealed a tonic pupil with an exaggerated pupillary constriction to dilute pilocarpine OD. Dilated exam revealed 2-3+ nuclear and cortical lens changes bilaterally. The patient's active lifestyle, personality, and biometry measurements made her a good candidate for multifocal IOL (MfIOL) implantation OS. Femtosecond laser-assisted cataract extraction with a ReSTOR +3 ADD (SN60D1) implantation was performed OS. Monofocal lens implantation (SN60WF) was performed OD 6 months later. Results: One year postoperatively, our patient had an uncorrected visual acuity (VAsc) of 20/15 for distance and J10 for near OD. Her VAsc was 20/25+1 for distance and $\mathrm{J} 1$ for near OS. Visual acuity when using both eyes was 20/15 for distance and J1 for near. Conclusion: Optimizing success for $\mathrm{MflOL}$ implantation is a multifactorial process. Large pupils preoperatively are of particular concern, as this may lead to increased dysphotopsia with pupil-dependent MfIOLs. Thus, patients with unilateral mydriasis, such as Aide's pupil, may have a beneficial outcome combining multifocal-monofocal implantation after bilateral cataract extraction, especially if they are not a candidate for monovision but desire spectacle independence.

(C) 2018 The Author(s)

Published by S. Karger AG, Basel
\end{abstract}




\section{Introduction}

The art of cataract surgery has shifted dramatically since its early inception, involving more surgical precision with higher patient expectations. Cataract surgery is becoming a refractive surgical procedure with greater importance on intraocular lens (IOL) calculations and selection for optimal results. IOL choices often range from monofocal IOLs to multifocal IOLs (MfIOLs). Monofocal lenses are designed to achieve an optimal vision for a specific target distance determined by the patient and surgeon. Visual acuity from a monofocal IOL is typically chosen for distance vision, whereas a MfIOL can give a range of vision, encompassing both near and distance. Thus, some patients with an active lifestyle are often more motivated to receive MfIOL implants in order to become spectacle independent for their daily activities.

MfIOLs provide a wider range of vision compared to monofocal implants due to their methods of light dispersion. In general, incoming light is separated into differing focal points, allowing for a range of focused vision [1,2]. MfIOLs can be refractive, diffractive, or a hybrid in regard to light dispersion. In hybrid diffractive-refractive MfIOLs such as ReSTOR (ALCON, Fort Lauderdale, FL, USA), apodization of the central diffractive zone results in a gradual transition of steps in the width and height from the center to the periphery of the lens. The gradual transition results in optical focal points of near, intermediate, and far vision [3]. However, apodization utilizes the changing pupil diameter to vary the amount of light on different focal points. For example, in low light, the patient's pupil enlarges, resulting in more light being refracted to the distance focus [4].

Therefore, patients receiving pupil-dependent MfIOLs are often evaluated for the quality and size of their preoperative pupil in both light and dark conditions. Dilated tonic pupil, also known as Adie's tonic pupil, is a condition which results in a large pupil which often contains segmental palsy of the iris sphincter [5]. Aide's pupil has a prevalence of $2 \%$, with a majority occurring unilaterally in women in their third decade [5]. However, to the best of our knowledge, MfIOL use in patients with unilateral Adie's pupil has not been well described.

We present our treatment of a patient with bilateral cataracts who desired to be spectacle independent despite a unilateral Adie's pupil. Optimal visual acuity was achieved by incorporating both a monofocal IOL and a MfIOL. Reviewing the literature has not revealed any successful reports of integrating monofocal and multifocal lens implantation in a patient with pupil abnormalities.

\section{Case Presentation}

A 74-year-old woman presented to our institution complaining of worsening near vision. The patient denied any previous eye history or relevant medical history. Corrected visual acuity was 20/30 in the right eye (OD) and 20/40 in the left eye (OS). Autorefraction revealed $+2.00-1.00 \times 104$ OD and $+1.25-0.75 \times 73$ OS.

Gross examination revealed a 6-mm pupil OD and a 5-mm pupil OS in low light. Anisocoria was greater with room light, demonstrating $6 \mathrm{~mm}$ OD and $3 \mathrm{~mm}$ OS. Slit lamp examination revealed a tonic pupil OD. In the left eye, a $5-\mathrm{mm}$ pupil briskly reacted to $3 \mathrm{~mm}$ with light. Topical dilute pilocarpine $(0.125 \%)$ demonstrated greater pupillary constriction OD compared to OS, consistent with Adie's tonic pupil.

Punctate staining on the inferior third of the cornea was present with decreased tear film in both eyes (OU). Intraocular pressure was $20 \mathrm{OD}$ and $17 \mathrm{OS}$. Dilated fundus exam revealed 2-3+ nuclear sclerosis and anterior cortical changes bilaterally. The vitreous was clear with 
the optic nerve, macula, and peripheral retina within normal limits. The patient was started on artificial tears three times a day and cyclosporine $0.05 \%$ twice a day.

The patient returned a month after the first visit for evaluation of keratoconjunctiva sicca and posterior chamber IOL (PC-IOL) calculation for cataract surgery. The patient desired to be free of glasses for her daily activities of reading, driving, and exercise. She also had an easygoing personality and was not concerned with driving at night. Thus, MfIOLs were considered. Corneal topography and optical biometry displayed normal Ks with a small amount of regular with the rule of astigmatism OU. Normal axial length and corneal thickness were also found OU. Slit lamp examination revealed resolution of punctate staining.

Two weeks after the IOL calculation, the patient was scheduled for cataract extraction OS with femtosecond-assisted laser (LensX; ALCON) combined with a ReSTOR +3 ADD (SN60D1) multifocal PC-IOL implantation. A target of $-0.50 \mathrm{D}$ was selected to achieve good near vision and guard against postoperative hyperopia. On postoperative day 1, her uncorrected visual acuity (VAsc) was 20/60 -2 for distance and $20 / 30$ for near OS. Two weeks postoperatively, her VAsc was 20/40 -1 for distance and 20/30 for near OS.

The surgery OD was performed 7 months after the first eye. A monofocal (SN60WF) PCIOL with a refractive target of $0.00 \mathrm{D}$ was chosen for better distance vision in the dominant eye. Standard phacoemulsification and extraction was performed. On postoperative day 1 , her VAsc was 20/30 - for distance and J10 for near OD. Two weeks postoperatively, her VAsc was 20/30 for distance and J10 for near OD.

One year postoperatively, our patient's VAsc was 20/15 for distance and J10 for near OD. VAsc $20 / 25+1$ for distance and J1 for near OS. Visual acuity OU was $20 / 15$ for distance and J1 for near (Fig. 1). Overall, the patient was very satisfied with the outcome of surgery and IOL selection, achieving her goal of spectacle independence without creating visual aberrations (e.g., glare or halo). Also, no exacerbation of dry eye disease had occurred following cataract surgery.

\section{Discussion}

Determining the right candidate for MfIOL implantation is an art that often relies on multiple factors. Understanding the patient's lifestyle requirements, visual goals, and personality traits are very important in determining candidacy for multifocal selection [6,7]. Patients who are highly motivated for complete spectacle independence, able to accept a small compromise in distance vision, and convey a relaxed personality are often more successful with multifocal implantation [7]. Personality type is often correlated with successful neuroadaptation and the subsequent reduction of postoperative dysphotopsias (e.g., glare and halos). As a result, lifestyle and vision questionnaires have become common tools used to assess the traits and goals of the patient.

Our patient had an active lifestyle. She was very motivated to carry out her activities without the aid of glasses. She also demonstrated a relaxed personality with realistic visual goals. Overall these attributes made her initially a strong candidate for MfIOL implantation.

However, the clinical presentation also plays a role in determining the type of IOL. It has been reported that patients with reduced contrast sensitivity [8] or pupil abnormalities, including eccentric pupil or iris coloboma $[9,10]$, may not be optimal candidates. Multifocal implantation may significantly decrease contrast sensitivity due to the IOL's light dispersion mechanics, especially in low mesopic conditions (low but not quite dark lighting situations) [11]. 
Thus, patients who have compromised contrast sensitivity, such as advanced glaucoma [12] or maculopathies, may be contraindicated for MfIOL use.

The size, shape, and functionality of the pupil are also important for MfIOL success. Patients with large pupils, typically larger than $5 \mathrm{~mm}$, are at increased risk for dysphotopsia postoperatively [9]. de Vries et al. [9] demonstrated that pupils which were $5.18 \pm 1.00 \mathrm{~mm}$ tended to have more difficulty reading in low mesopic conditions. These patients were also more likely to become dissatisfied with their overall visual outcome [9]. ReSTOR, a hybrid refractive-diffractive apodized MfIOL, are pupil-dependent lenses. Based on the size of the pupil, more light is directed to either the distant or near focal point. Large pupils direct a higher proportion of light to the distant focal point under low mesopic conditions, which may cause visual impairments for patients desiring near vision in low light settings [4]. Thus, preoperative consideration of pupil size may be very important, especially in pupil-dependent MfIOL.

Our patient presented with a 6-mm fixed pupil. Therefore, she was not an ideal candidate for pupil-dependent MfIOL, such as ReSTOR, due to an increased risk of dysphotopsia. However, her left eye had a normal, responsive pupil. Personality, lifestyle, and biometry measurements made her a valid candidate for MfIOL OS, while a non-pupil-dependent lens was more suitable OD. We have had good visual outcomes mixing IOL types in previous patients. Thus, the idea of combining multifocal-monofocal implantation was deemed as a potential option.

The effectiveness of unilateral implantation of MfIOL during bilateral cataract extraction has been demonstrated. Cionni et al. [13] performed a 6-month prospective study comparing patient satisfaction and spectacle independence for unilateral versus bilateral implantation of ReSTOR (SN60D1) MfIOLs. Patients with multifocal-monofocal implantation achieved a 75\% satisfaction and $65 \%$ degree of spectacle independence. Although lower than bilateral multifocal implantation, there was no statistical difference between unilateral use [13]. Our patient gained complete spectacle independence when combining multifocal-monofocal implantation. At one year postoperatively, visual acuity was 20/15 for distance and J1 for near when using both eyes. Thus, unilateral multifocal implantation for bilateral cataract extraction may be a viable option, especially when physiologic conditions such as reduced contrast sensitivity or pupil abnormalities exist in one eye.

Other surgical options were considered for this patient such as monovision and pupil nondependent MfIOL. A comparison of monovision to diffractive MfIOL has demonstrated similar results regarding visual acuity and spectacle independence [14]. However, the patient's lack of previous experience with monovision made this less likely of a possibility. Alternatively, pupil non-dependent MfIOLs were not considered as our surgeon had more experience and better visual outcomes using ReSTOR.

In summary, optimizing success for MfIOL implantation is a complex, multifactorial process. Many factors such as patient expectation, personality traits, and clinical presentation may affect the overall visual outcome and satisfaction of the patient. For one, large pupils preoperatively are of particular concern as this may lead to an increase in dysphotopsia, especially in low mesopic conditions with pupil-dependent MfIOLs. However, patients with unilateral mydriasis may not be contraindicated for MfIOL implantation in the unaffected eye. In our patient, combining multifocal-monofocal implantation after bilateral cataract extraction resulted in an excellent visual outcome and patient satisfaction. Monofocal-multifocal implantation may be beneficial for patients who are not a candidate for bilateral MfIOL or monovision, but desire to become spectacle independent. 


\section{Statement of Ethics}

The authors did not have any ethical conflicts to disclose.

\section{Disclosure Statement}

The authors did not report any potential conflict of interests.

\section{Funding Sources}

No external funding/grants.

\section{References}

1 Alfonso JF, Fernández-Vega L, Baamonde MB, Montés-Micó R. Prospective visual evaluation of apodized diffractive intraocular lenses. J Cataract Refract Surg. 2007 Jul;33(7):1235-43.

2 Zhao G, Zhang J, Zhou Y, Hu L, Che C, Jiang N. Visual function after monocular implantation of apodized diffractive multifocal or single-piece monofocal intraocular lens Randomized prospective comparison. J Cataract Refract Surg. 2010 Feb;36(2):282-5.

3 Davison JA, Simpson MJ. History and development of the apodized diffractive intraocular lens. J Cataract Refract Surg. 2006 May;32(5):849-58.

4 Alfonso JF, Fernández-Vega L, Baamonde MB, Montés-Micó R. Correlation of pupil size with visual acuity and contrast sensitivity after implantation of an apodized diffractive intraocular lens. J Cataract Refract Surg. 2007 Mar;33(3):430-8.

5 Thompson HS: Segmental palsy of the iris sphincter in Adie's syndrome. Arch Ophthalmol. 1978;96: 1615-20.

6 Alio JL, Plaza-Puche AB, Férnandez-Buenaga R, Pikkel J, Maldonado M. Multifocal intraocular lenses: an overview. Surv Ophthalmol. 2017 Sep - Oct;62(5):611-34.

7 Braga-Mele R, Chang D, Dewey S, Foster G, Henderson BA, Hill W et al.; ASCRS Cataract Clinical Committee. Multifocal intraocular lenses: relative indications and contraindications for implantation. J Cataract Refract Surg. 2014 Feb;40(2):313-22.

8 Woodward MA, Randleman JB, Stulting RD. Dissatisfaction after multifocal intraocular lens implantation. J Cataract Refract Surg. 2009 Jun;35(6):992-7.

9 de Vries NE, Webers CA, Touwslager WR, Bauer NJ, de Brabander J, Berendschot TT et al. Dissatisfaction after implantation of multifocal intraocular lenses. J Cataract Refract Surg. 2011 May;37(5):859-65.

10 Cerviño A, Hosking SL, Montés-Micó R, Alió JL. Retinal straylight in patients with monofocal and multifocal intraocular lenses. J Cataract Refract Surg. 2008 Mar;34(3):441-6.

11 Bühren J, Terzi E, Bach M, Wesemann W, Kohnen T. Measuring contrast sensitivity under different lighting conditions: comparison of three tests. Optom Vis Sci. 2006 May;83(5):290-8.

12 Kumar BV, Phillips RP, Prasad S. Multifocal intraocular lenses in the setting of glaucoma. Curr Opin Ophthalmol. 2007 Feb;18(1):62-6.

13 Cionni RJ, Osher RH, Snyder ME, Nordlund ML. Visual outcome comparison of unilateral versus bilateral implantation of apodized diffractive multifocal intraocular lenses after cataract extraction: prospective 6month study. J Cataract Refract Surg. 2009 Jun;35(6):1033-9.

14 Stock RA, Thumé T, Paese LG, Bonamigo EL. Subjective evaluation of uncorrected vision in patients undergoing cataract surgery with (diffractive) multifocal lenses and monovision. Clin Ophthalmol. 2017 Jul;11:1285-90. 


\section{Case Reports in Ophthalmology}
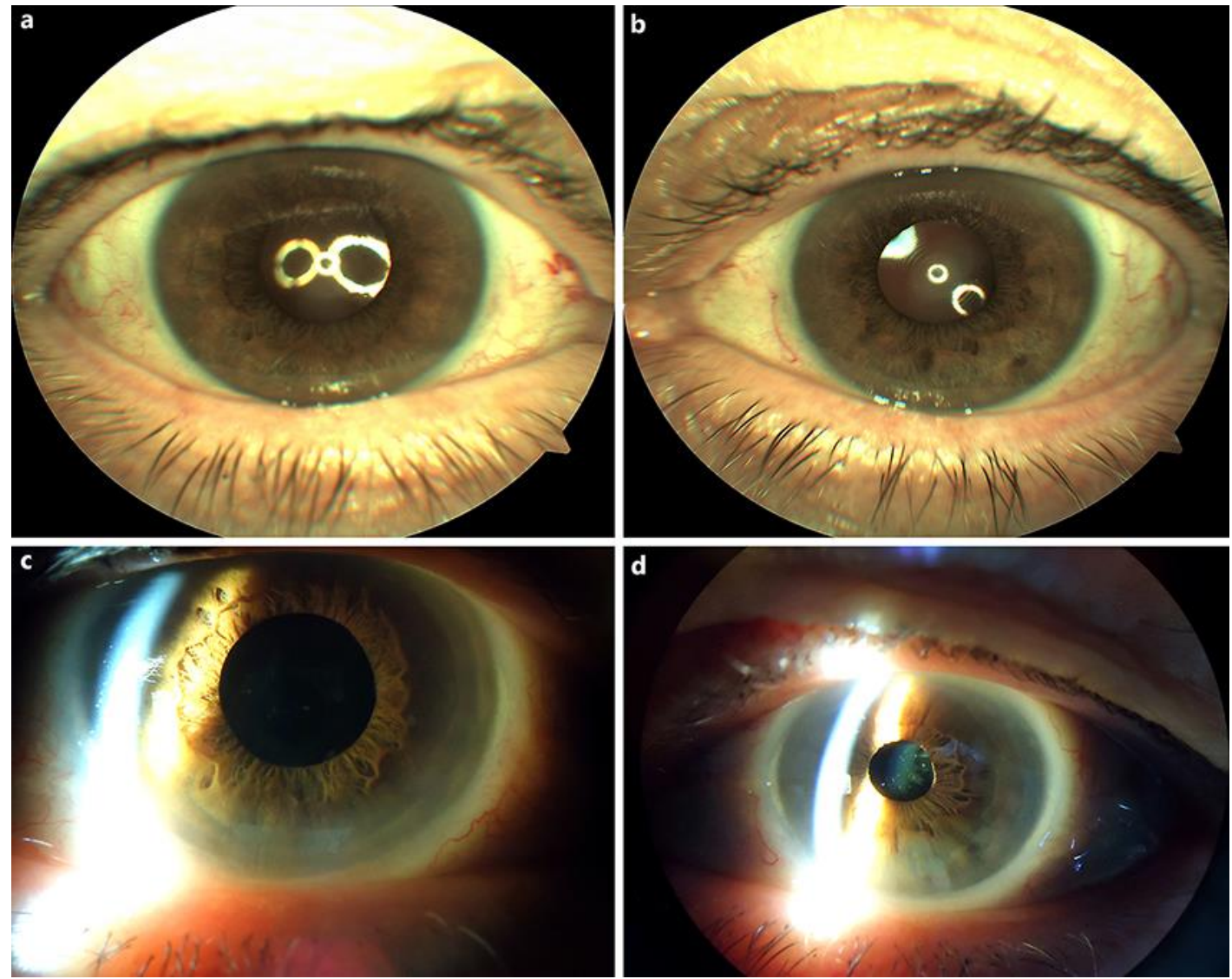

Fig. 1. Imaging of right and left eye one year after bilateral cataract extraction. a A 6-mm pupil is noted with a monofocal (SN60WF) posterior chamber intraocular lens (PC-IOL) on the right eye (OD), performed with a fundus camera at low light. b A 5-mm pupil is noted with ReSTOR +3 ADD (SN60D1) multifocal PC-IOL implantation with visible concentric rings on the left eye (OS), performed with a fundus camera at low light. c Slit lamp examination noting a tonic, dilated pupil with no visible sphincter contraction OD using direct light. $\mathbf{d}$ A regular, brisk pupillary reaction to $3 \mathrm{~mm}$ with direct light OS. 